\title{
A rational fraction polynomials model to study vertical dynamic wheel-rail interaction
}

\author{
N. CORREA, E. G. VADILLO, J. SANTAMARIA, J. GOMEZ
}

Department of Mechanical Engineering. University of the Basque Country UPV/EHU. Alameda Urquijo s.n., 48013 Bilbao, Spain

\begin{abstract}
NOTICE: This is an electronic version of an article published in Journal of Sound and Vibration, Vol. 331, pp. 1844-1858. 2012. Changes resulting from the publishing process, such as editing, corrections, structural formatting, and other quality control mechanisms may not be reflected in this document.

JOURNAL OF SOUND AND VIBRATION is available online and the final version can be obtained at:

http://dx.doi.org/10.1016/j.jsv.2011.12.012
\end{abstract}

DOI: $10.1016 /$ j.jsv.2011.12.012

Please cite this paper as: Correa, N., Vadillo, E.G., Santamaria, J., Gomez, J.

A rational fraction polynomials model to study vertical dynamic wheel-rail interaction. Journal of Sound and Vibration, Vol. 331, pp. 1844-1858. 2012

Corresponding author. Email: ernesto.garciavadillo@ehu.es 


\title{
A rational fraction polynomials model to study vertical dynamic wheel-rail interaction
}

\author{
N. Correa, E. G. Vadillo*, J. Santamaria, J. Gómez \\ Mechanical Engineering Department, University of the Basque Country UPV-EHU, \\ Escuela Técnica Superior de Ingeniería, Alda Urquijo s/n, 48013 Bilbao, Spain
}

\section{Summary}

This paper presents a model designed to study vertical interactions between wheel and rail when the wheel moves over a rail welding. The model focuses on the spatial domain, and is drawn up in a simple fashion from track receptances. The paper obtains the receptances from a full track model in the frequency domain already developed by the authors, which includes deformation of the rail section and propagation of bending, elongation and torsional waves along an infinite track. Transformation between domains was secured by applying a modified rational fraction polynomials method. This obtains a track model with very few degrees of freedom, and thus with minimum time consumption for integration, with a good match to the original model over a sufficiently broad range of frequencies. Wheel-rail interaction is modelled on a non-linear Hertzian spring, and consideration is given to parametric excitation caused by the wheel moving over a sleeper, since this is a moving wheel model and not a moving irregularity model. The model is used to study the dynamic loads and displacements emerging at the wheel-rail contact passing over a welding defect at different speeds.

Keywords: wheel, rail, track, welding defects, Rational Fraction Polynomials

\section{Introduction}

The presence of welds between rail sections on track with continuous welded rail (CWR) frequently causes irregularity in the geometry of the rail around the weld. Although this irregularity is mellowed by the grinding process applied to rails after welding, normally a certain amount of impairment remains, which is restricted by acceptance regulations for rail welds in legislation prevailing in each country. Irregularity on the railhead surface may considerably increase dynamic loads during contact as the wheel moves over the rail, and this could damage the track.

\footnotetext{
${ }^{*}$ Corresponding author. Tel.: +34 94601 4223; fax: +34 946014215.

E-mail address: ernesto.garciavadillo@ehu.es (E.G. Vadillo).
} 
The acceptability of rail welds has been widely studied in [1,2]. These articles present one criterion based on the gradient of the rail surface in the area the geometry of which is affected by the weld, and another based on its curvature. This advanced criterion showed that welds which were acceptable in accordance with normal regulations could be unacceptable if the new criterion were taken into account. International regulations nevertheless continue to impose restrictions exclusively on maximum deviation on the defect and its planitude [3].

In general, two different methods may be used to study wheel-rail interaction: in the frequency domain or in the spatial domain. Each contains a number of applications that present certain advantages with respect to the other.

Working in the frequency domain permits a study of the vehicle-track interaction and also enables the steady-state response to be obtained in an accurate manner and with low computing time expenses, both for tracks with discrete sleepers [4] and for continuously supported track [5]. The utilisation of frequency domain models makes it possible to obtain linear models which describe the real track dynamic behaviour to a great degree of precision, including rail transversal cross-section deformation and elongation, flexion and torsional wave propagation along the infinite track [6-8]. The ease of calculation using frequency domain models also enables the optimisation of track parameters in order to improve their behaviour [9].

In non-linear and non-steady-state problems, however, as is the case in this work, timedomain models must be employed. These require more time for calculation, even with models much less accurate than those which may be secured by working in the frequency domain.

Thus this paper has chosen to develop a time domain track model obtained from the transformation of a comprehensive frequency domain model able to study medium-high frequency ranges developed by the authors [6-8], which enables the consideration of transversal cross-section deformation of an infinite rail, using a modified Rational Fraction Polynomials (RFP) method. This method availed itself of an extremely detailed model in the frequency domain to obtain a simplified spatial domain model valid up to frequencies as high as required for each particular problem.

Recently Mazilu has developed a model alternative to the one proposed in this paper, by using the Green's functions of the track to solve wheel/rail interaction problems, both linear [10] and also non-linear [11]. Such a method has been used to study the response to several defects such as rail corrugation and wheel flats, considering the rail as an infinite Timoshenko beam.

Alternatively, the method developed in this paper produces, as one of its main advantages, a very simple time domain model represented by a system of equations in the space domain with very few degrees of freedom, thus obtaining highly accurate results, the calculation of which entails an extremely low computational outlay. This method is based on the fitting of track receptances via transfer functions, with subsequent passage to the time domain. Therefore, the dynamic influence of pads, sleepers, ballast, etc. (including their masses, stiffnesses, dampings 
and moments of inertia) is taken into account through such track receptances. These types of transformation methods, for tracks with both continuous support and discrete support, have been applied by $\mathrm{Wu}$ and Thompson [12-18] and other researchers [19,20] to examine various phenomena observed in wheel/rail contact.

The paper focuses on a track with discrete support. As the rail vehicle runs along a discretely supported track, the track receptance below the wheel is changing with the position of the contact patch within a sleeper bay. Therefore the track receptances in the different rail sections along the sleeper bay must be calculated, and adjusted using the RPF. As a result, the coefficients of the system of differential equations that describes the track dynamics change at each instant in time (because the receptance is changing). The combination of the set of systems of differential equations for each section of the sleeper bay leads to a system of differential equations with coefficients that vary with distance along this sleeper bay. Track periodicity is introduced to the model at this point, by periodically repeating the coefficients of the system of equations (with a spatial period equal to the sleeper bay distance) in every bay travelled by the wheel. It is assumed, therefore, that all the track spans are identical.

The difficulty of studying a track with discrete support is due to the extreme variability of the track receptance along a span. By way of an example, Fig. 1 shows the receptances on certain sections of a span of a discretely supported track. The positions on the track span of those sections are shown in Fig. 2. The receptances were obtained from the track characteristics set out in Table 1. This shows the substantial difference between the various receptances, in particular at frequencies in proximity to the first vertical pinned-pinned mode around 1,080 Hz, with a midspan resonance and an anti-resonance over the sleeper. Receptances of sections located between midspan and over the sleeper take up an intermediate format between that of these two, in such a way that they show a resonance and an anti-resonance in close proximity to this frequency.

Using the RFP method also presents a special difficulty for some tracks with discrete support, depending on the particular parameters of the track, including its stiffness and damping values. The novelty of this method lies in the combined use of the RFP together with optimisation methods based on multi-objective genetic algorithms. This has two advantages. Firstly, it makes it possible to easily and more accurately adjust track receptances. Secondly, it improves the integration of the system of differential equations obtained from the application of the RFP method to describe the track. In addition to application of the genetic algorithm method, the paper also presents a number of fitting methods that have been tested as alternatives to obtain the time domain system. 

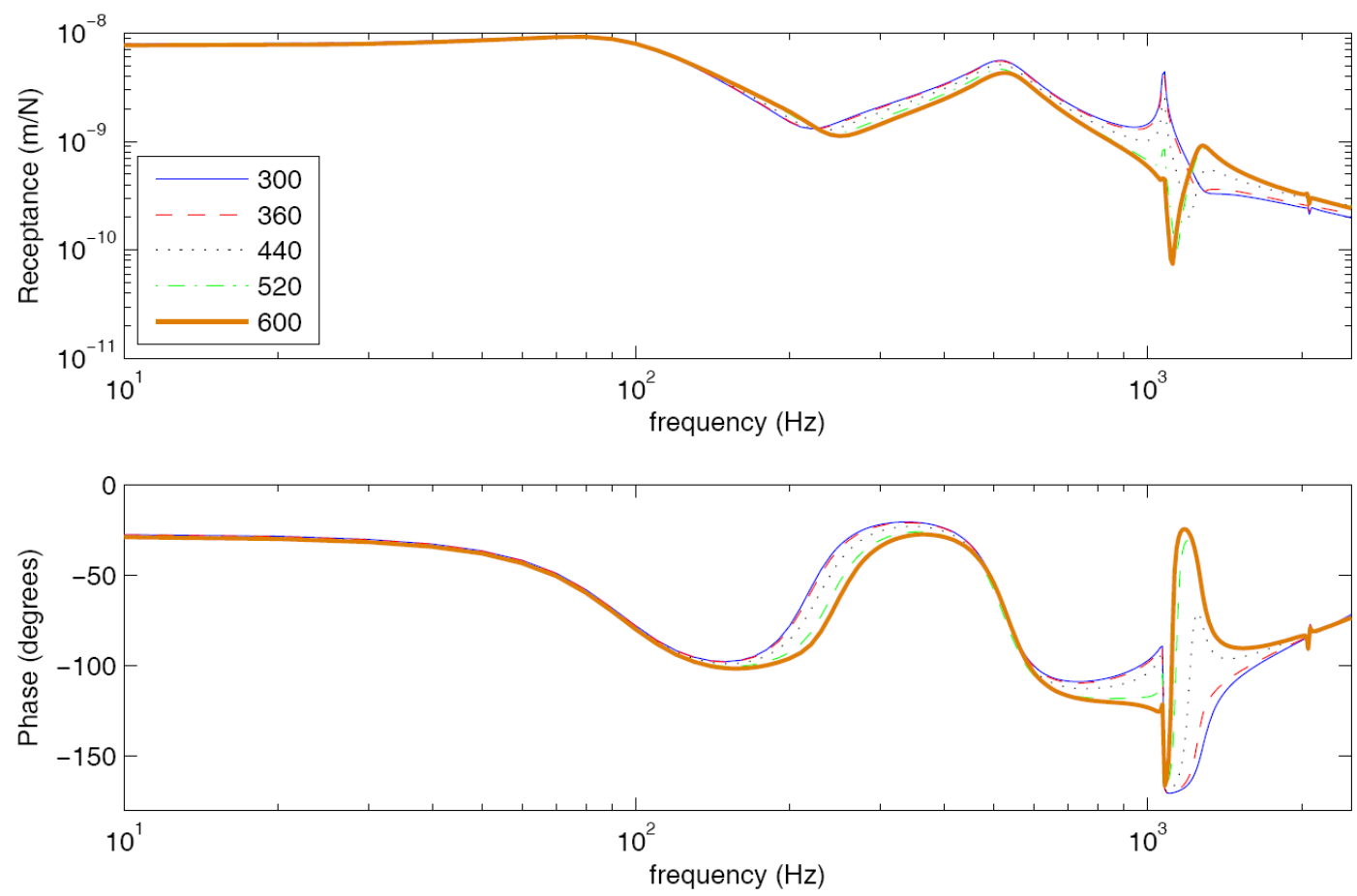

Fig. 1. Track receptances on span sections at millimetres: 300, 360, 440, 520, 600.

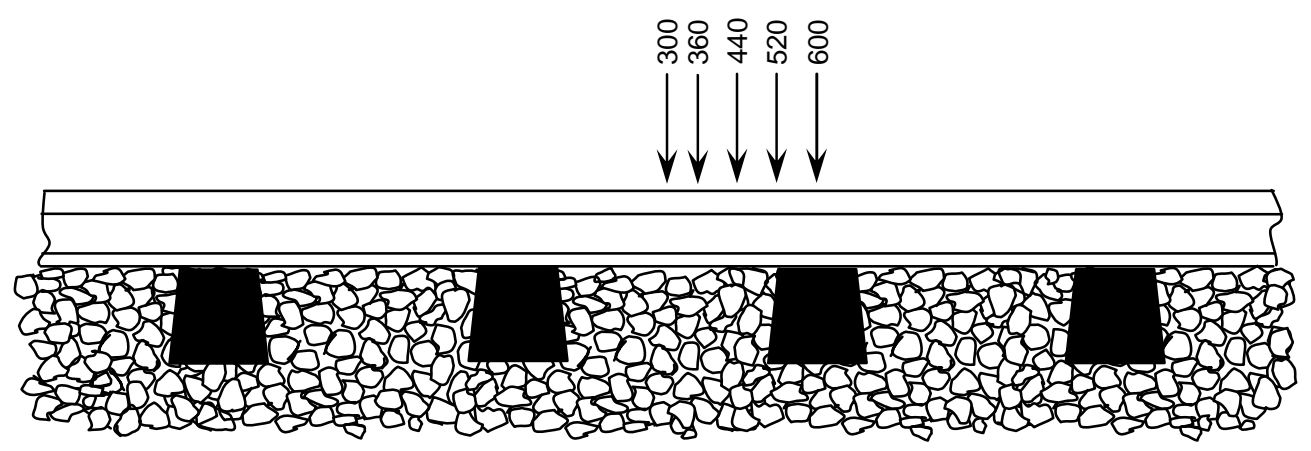

Fig. 2. Location of span sections at millimetres: 300, 360, 440, 520, 600.

For the sake of simplicity the wheel has been modelled as a mass over which the corresponding weight of the vehicle is applied, and contact has been represented by a non-linear Hertzian spring, in such a way as to permit loss of contact between the wheel and the rail that may occur when the wheel moves over the welding. Moreover, considering that it is the wheel which moves and not the irregularity, it is possible to take account of the excitation at the sleeper-passing frequency. 
Table 1: Track parameters

\begin{tabular}{|l|c|}
\hline Pad stiffness $(\mathrm{kN} / \mathrm{mm})$ & 348.6 \\
\hline Pad damping (-) & 0.29 \\
\hline Ballast stiffness (kN/mm) & 50 \\
\hline Ballast damping (-) & 1 \\
\hline Sleeper mass $(\mathrm{kg})$ & 324 \\
\hline Sleeper spacing $(\mathrm{m})$ & 0.6 \\
\hline Rail & $60 \mathrm{E} 1$ \\
\hline
\end{tabular}

The interaction model presented in this paper is applied to the study of the dynamic response of the wheel and the rail when the former has a wheel flat, and also when the latter has an acceptable weld pursuant to the criteria of regulations [3]. This study is performed for wheelsets travelling at different speeds.

\section{Track model developed}

\subsection{Rational fraction polynomials method applied to the case of the track}

The model developed uses the rational fraction polynomials method [21]. This method obtains in the s-plane (Laplace plane) the transfer functions related to the receptances which define the dynamic system studied (the track, in our case) for a specific frequency range. These $G(s)$ transfer functions are expressed as polynomial quotients (Eq. (1)), the coefficients of which are calculated by solving the problem of minimising Eq. (2). This corresponds to minimisation of the quadratic error between the original receptance and the receptance fitted during optimisation in the frequency range within which the fitting is being performed.

$$
G(s)=\frac{Y(s)}{F(s)}=\frac{B(s)}{A(s)}=\frac{b_{1} s^{m}+b_{2} s^{m-1}+\ldots+b_{m-1} s+b_{m}}{s^{n}+a_{1} s^{n-1}+a_{2} s^{n-2}+\ldots+a_{n-1} s+a_{n}}
$$

In Eq. (1), $Y(s)$ is the Laplace transform of the displacement function of the point of the rail in contact with the wheel, $F(s)$ is the Laplace transform of the contact force, and $A(s)$ and $B(s)$ the denominator and numerator of the transfer function.

In Eq. (2), $\mathbf{h}$ is the track receptance which is being fitted, $\boldsymbol{\omega}$ is the vector of angular frequencies, length $p$, and wt is a weight vector for error at the various frequencies at which the fitting is being performed.

$$
\min _{a, b} \sum_{k=1}^{p} \mathbf{w t}(k)\left|\mathbf{h}(k)-\frac{B(\boldsymbol{\omega}(k))}{A(\boldsymbol{\omega}(k))}\right|^{2}
$$


The degrees of the numerator and denominator polynomials $m$ and $n$ of the transfer functions arising from Eq. (1) match the number of zeros and poles of these transfer functions, respectively.

When the transfer function has been calculated on different sections of the track span, the associated system of differential equations is obtained via the inverse Laplace transform of the transfer function. The result of this operation is an ordinary differential equation of an order equal to the order of the denominator polynomial of the transfer function (3), where $y$ is the displacement of the point of the rail in contact with the wheel and $f$ is the contact force. Eq. (3) may be transformed into a first-order system of differential equations (4).

$$
\begin{aligned}
& y^{n)}(t)+a_{1} y^{n-1)}(t)+a_{2} y^{n-2)}(t)+\ldots+a_{n-1} y^{\prime}(t)+a_{n} y(t)= \\
& b_{1} f^{m)}(t)+b_{2} f^{m-1)}(t)+\ldots+b_{m-1} f^{\prime}(t)+b_{m} f(t) \\
& x_{1}^{\prime}(t)=x_{2}(t)+c_{1} f(t) \\
& x_{2}^{\prime}(t)=x_{3}(t)+c_{2} f(t) \\
& \ldots \\
& x_{n-1}^{\prime}(t)=x_{n}(t)+c_{n-1} f(t) \\
& x_{n}^{\prime}(t)=-a_{n} x_{1}(t)-a_{n-1} x_{2}(t)-a_{n-2} x_{3}(t)-\ldots-a_{1} x_{n}(t)+c_{n} f(t)
\end{aligned}
$$

where the values corresponding to $c$ are [22]:

$$
\begin{aligned}
& c_{1}=b_{1} \\
& c_{2}=b_{2}-a_{1} b_{1} \\
& c_{3}=b_{3}-\left(a_{2} b_{1}+a_{1} b_{2}\right) \\
& \cdots \\
& c_{n}=b_{n}-\sum_{k=1}^{n-1} a_{n-k} c_{k}
\end{aligned}
$$

and thus:

$$
y(t)=x_{1}(t)
$$

\subsection{Practical application of the fraction polynomials method to the track}

For the purposes of practical implementation of this method, there must be a constant guarantee of reaching a stable system of differential equations. Obviously an unstable system would serve no purpose. In order to achieve stability, it must be ensured that the poles of the transfer function denominator have a negative real part. Thus it is necessary to add the constraint to the problem of minimising Eq. (2) that the transfer function poles must be stable. 
It was observed in the course of this process that it is important that a suitable number of zeros and poles be used for the fitting. An excessively small number of poles and zeros would lead to a poor receptance fit, and could even produce a resultant transfer function the associated receptance of which shows fewer resonances or anti-resonances than the original receptance. On the other hand, contrary to what might be assumed initially, too many poles and zeros do not furnish a satisfactory solution either, since an excessive number would force the excess poles and zeros to cancel each other out during optimisation in order to perform a satisfactory fit of the receptances [23]. This could lead to numeric problems during integration, and could even entail the emergence of resonances and anti-resonances in the fitted transfer function that do not actually exist.

It must also be considered that, when the wheel moves over the track, the track receptance at the point of contact varies, and thus the fitting must be carried out using the RFP method for different sections of the track span. Then, for each span location where the adjustment is carried out, different values for $a_{i}$ and $b_{i}$ are obtained, which lead to a different system of equations for each span location. It is assumed that all the spans in the track are identical, and therefore that the receptance in each location within the span is the same for such location in all the spans. As a consequence, the coefficients for $a_{i}$ and $b_{i}$ are repeated periodically for each span along the track. This leads to a system of differential equations with variable coefficients in space. In those locations within the span where no adjustment has been carried out using the RFP method, the coefficients for $a_{i}$ and $b_{i}$ are calculated by means of linear interpolation from those coefficients corresponding to the two closest locations where the adjustment has been actually carried out.

The fact that different coefficients in space are included in the system of equations adds another difficulty to the problem in that the coefficients of the polynomials obtained from the receptance fit on each section of the span must be of a similar order of magnitude, since otherwise there could be abrupt variations in space of the system of differential equations, which not only do not have any physical meaning, but would also considerably hamper the integration of the system of equations with variable coefficients.

\subsection{Optimisation in the rational fraction polynomials method}

In order to carry out the optimisation expressed in Eq. (2), tests were conducted with a number of algorithms, the advantages and disadvantages of which are discussed below: 


\subsubsection{Optimisation using sequential quadratic programming methods (SQP)}

This approaches the objective function and the constraints of the problem using quadratic functions around the point at which the solution is located in each iteration during optimisation. This is an optimisation with a single objective function, that shown in Eq. (2), in which the errors made in the fittings have been separated into real part and imaginary part, since the objective function must be real, and not complex. The variables are the coefficients of the numerator polynomial of transfer function $\left(b_{i}\right)$ and the real parts $\left(r_{i}\right)$ and imaginary parts $\left(n_{i}\right)$ of the poles of the transfer function (see Eq. 4). The constraints are that the real part of the poles must be negative $\left(r_{i}<0\right)$. The sum of quadratic errors at each frequency between the real and imaginary parts of the real and fitted receptance is minimised, and subsequently the totals of the quadratic errors are added together. This method has also been tested in two ways:

a. Calculating the derivatives in numeric fashion, from evaluations of the objective function.

b. Entering the derivatives of the objective function in analytic fashion.

This method is programmed in the MATLAB optimisation toolbox [24], in the fmincon function.

The results obtained through this method were not satisfactory. This was because it is an extremely local method, and the objective function employed has a large number of relative minima, and thus in all cases a local minimum was obtained that did not secure a proper fit for the receptance. Finding a solution would have necessitated taking an initial solution in very close proximity to the global minimum, which in principle is unknown, and therefore many initial solutions would had to have been taken, sweeping across an extremely wide space. This did not serve to solve the problem envisaged.

\subsubsection{Optimisation using the Nelder-Mead algorithm}

This method enables optimisation of the function with no need to know its derivatives in analytic fashion, and is based solely on evaluations of the function.

The objective function, the constraints and the variables optimised are the same as in the preceding case.

It is programmed in MATLAB in the fminsearch function [24], modified so as to allow constraints to be entered.

As in the case of SQP, it is a local algorithm and does not locate the solution with ease. It has also been observed that it is only possible to work in a practical manner with very few variables to carry out the optimisation. 


\subsubsection{Optimisation using genetic algorithms, with a single objective function}

These methods do not require the derivatives of the objective function to be known. The process is based on the evolution of an initial population, generation after generation, through the selection, reproduction, crossover and mutation procedures. It is a global method that does not require an initial approach in close proximity to the solution.

It is programmed in MATLAB with the $g a$ function in the Global Optimisation Toolbox [25].

It has been used in two different ways:

a. With the same variables, objective function and constraints as those used in the preceding cases. It required an extremely large initial population for good results to be achieved, and a considerable period for calculation. Practical utilisation of the method requires a large memory, and it is therefore unfeasible.

b. Carrying out minimisation using the damped Gauss-Newton method (as programmed in the MATLAB invfreqs function [26]) to obtain the coefficients of the transfer function polynomials, and forcing pole stability by changing the sign of the real part of the poles in cases where this is positive. The process commences again with this new value as the initial solution to the Gauss-Newton problem, and so on. In this case a weight vector is added to each frequency in which the receptance is fitted. The aim of this vector is to increase the importance of some frequencies over others when optimisation is carried out, and this proved extremely effective in securing a satisfactory fit. With this method, optimisation with genetic algorithms is used precisely to calculate the optimum weight vector for the best possible fit. In this case the objective function is the same as in the preceding examples, although the weights are entered at each frequency, in such a way that what is minimised is the weighted sum of quadratic errors at each frequency, between the original transfer function and the fitted function. The variables are the components of the weight vector, and the constraints are that these weights must have values of between 0 and 1 .

This algorithm has one marked disadvantage: the large number of unknowns (as many as frequency lines), and this makes it extremely difficult to achieve a good solution within a short space of time. Moreover, the transfer function coefficients obtained using this method have excessively high values, showing major variations with respect to those obtained on adjacent sections of the span, and this poses difficulties in terms of integration.

\subsubsection{Optimisation using multi-objective genetic algorithms}

As in the preceding case, these are global methods that do not require the derivatives of the objective function to be known. They are based on the evolution of an initial population, generation after generation, through the selection, reproduction, crossover and mutation procedures. Unlike the preceding case, here there may be more than one objective function, and 
there is not only one solution but rather a Pareto front, which contains a set of non-dominated solutions.

Two objective functions are employed, as follows:

- The weighted sum of quadratic errors at each frequency in the fitting of the transfer functions.

- The magnitude of the coefficients of the transfer function fitted. This prevents excessively high coefficients from emerging, and also forces coefficients for the various sections of the track to be of the same order of magnitude.

The variables are the weights at each frequency. Here the process has also been deployed in two different ways:

a. With the weights as real variables, which also requires a considerable amount of time for calculations, as in the case of an objective function, though the former process secures better results since the coefficients are much more homogeneous among the adjacent sections.

b. With the weights as binary variables ( 0 or 1$)$. This substantially reduces calculation time since the number of values that the optimisation variables may take is finite, unlike the process carried out with real numbers. It also achieves some very good fitting results.

\subsubsection{Method finally developed}

From all the methods developed and tested the one chosen has been the method described just before. By way of a summary, this method consists of obtaining, for each section of the span on which receptance is being fitted, the weight vector that provides the best possible fit of the receptances by solving the problem defined in Eq. (2).

The algorithm used substantially reduces the calculation time required for fitting the receptances with respect to a direct application of the method used by previous researchers, and also ensures that the magnitude of the coefficients of the transfer function polynomials is set. This facilitates subsequent integration of the system of equations in the space domain shown in Eq. (4).

For the purposes of optimisation using genetic algorithms, the function already available in MATLAB known as gamultiobj [25], was used, with binary variables.

\subsection{Application of the transformation method to obtain the track model}

As a first step, the receptance of the specific track under study is measured or calculated. Next, the receptance curve-fitting specific for that track is undertaken. This section shows the fits carried out for some of the receptances on a track the parameters of which are shown in Table 1. These parameters lead to the same receptances shown in $[16,17]$, allowing therefore to 
validate results. These receptances were calculated, as already mentioned, using the first module of a mathematical tool developed by the authors [6-8].

In this case, receptances were calculated for track span sections with $4 \mathrm{~cm}$ interspacing. This distance is sufficient for an appraisal of variation in the receptances between sections, and is also short enough to properly register the effect of the parametric excitation that arises when the vehicle moves over each span of track - it has been ascertained that this is of great importance in connection with wheel-track interaction.

Fig. 3 shows the fittings of the receptance over the sleeper and at $320 \mathrm{~mm}$ from the sleeper respectively. It should be mentioned that, for this particular track parameters, securing a good receptance fit at all frequencies was a complicated process, especially at low frequencies and high frequencies. Though it may be tempting to forsake a satisfactory fit at low frequency and focus on medium and high frequencies, it should be borne in mind that the sleeper-passing frequency, which can be extremely high, occurs precisely at low frequency, except in the case of high-speed trains. Hence the preference in these examples in the figures towards a slightly impaired fit at medium frequency (in the case of the figures shown, around the first antiresonance) rather than low frequency. Furthermore, the error in an anti-resonance is shown as an exaggeration on the logarithmic scale representation. On the linear scale this error is completely negligible. With regard to the fitting of the phase of receptances, the method shows a limitation in that it forces the phase at frequency $0 \mathrm{~Hz}$ to equal $0^{\circ}$. As may be observed, the fit result is very good indeed. For other track parameters the fit result is even better.

When the transfer functions associated with the receptances of each section of the span have been obtained, the inverse Laplace transform is applied to them. The result, as stated above, is an ordinary differential equation of an order equal to the order of the denominator polynomial of the transfer functions, which may be moved to the same first-order number of equations. Thus a system of equations with variable coefficients in space is obtained (due to the various positions of the wheelset on the span of track).

The greatest difficulty encountered in this method for transfer to the spatial domain lies in obtaining such a system. Once it has been calculated, however, integration is very swift since the system approached has very few degrees of freedom $(2 n)$. It must be borne in mind that this calculation is performed only once and that, once the fit has been made, it is not necessary to repeat this unless any changes are made to the track. 
(a)

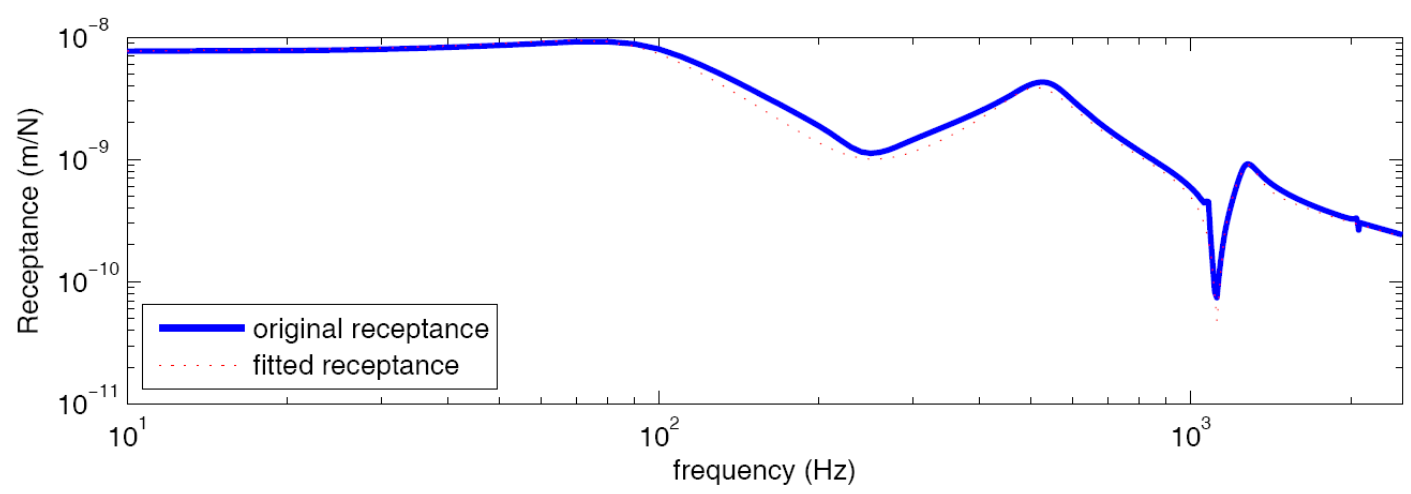

(b)

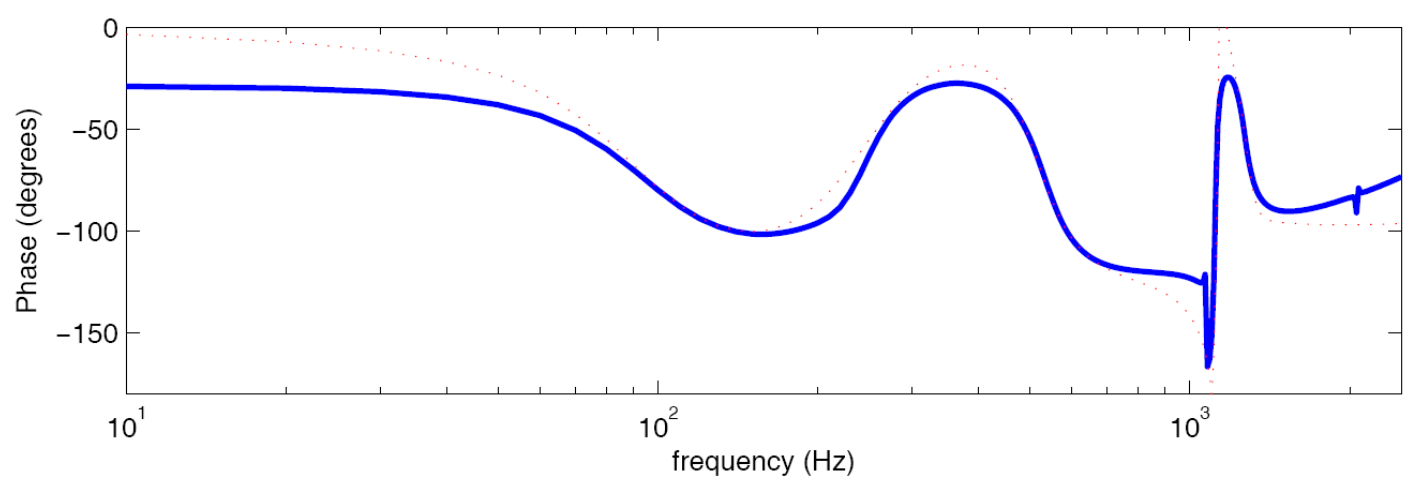

(c)

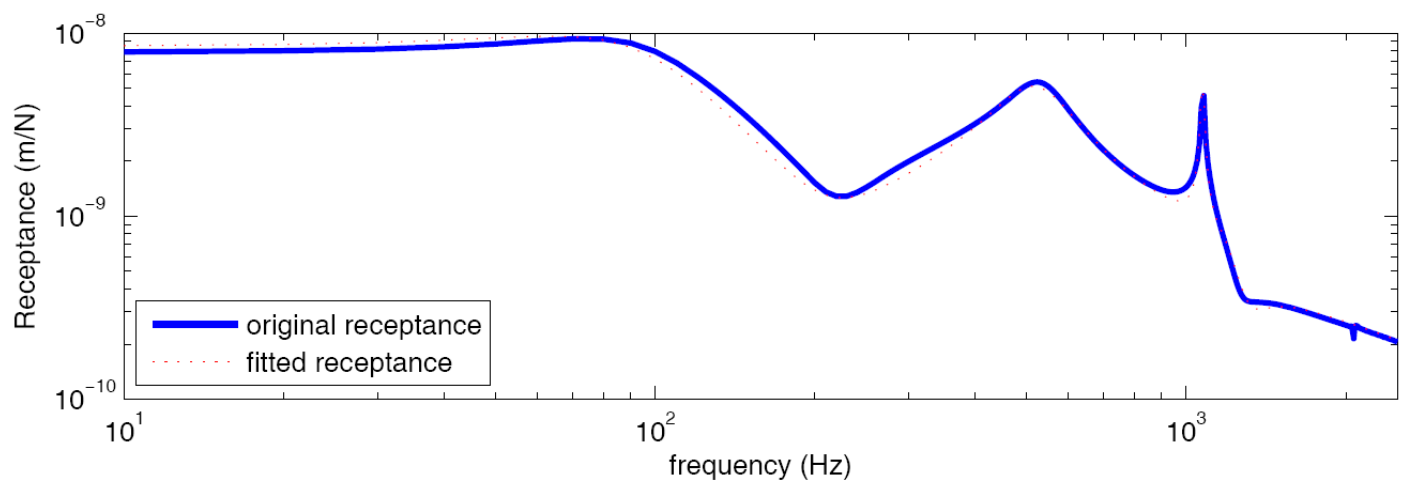

(d)



Fig. 3. Receptance fittings using the method developed on the sections located over the sleeper: (a) module, (b) phase; and at millimetre 320 of the span: (c) module, (d) phase. 


\section{Rail-wheel interaction}

The system of differential equations obtained in the preceding section is used to define the movement of the point on the rail located at the centre of the contact patch for its various positions along the rail.

Although the wheelset could be represented by a much more complex model, in this case it was decided to use a mass to which the total weight of the vehicle corresponding to this wheel is applied $(W)$. The equation would thus be as shown in Eq. (6).

$$
m_{w} \ddot{x}_{w}+f_{\text {contact }}=W
$$

where $x_{w}$ is the vertical displacement of the wheel and $m_{w}$ is its mass.

The contact force $f_{\text {contact }}$ between the wheel and the rail is described in the non-linear expression shown in Eq. (7).

$$
\begin{gathered}
f_{\text {contact }}=C_{H}\left(x_{w}-y-r\right)^{3 / 2} \text { when } x_{w}-y-r \geq 0 \\
f_{\text {contact }}=0 \text { when } x_{w}-y-r \leq 0
\end{gathered}
$$

where $y$ is the vertical displacement of the rail at the contact point, $C_{H}$ is the Hertz contact constant, and $r$ represents any irregularity of the rail or the wheel. If the irregularity is in the rail, if $r$ is positive this indicates a rail dip, whereas if it is negative this shows an asperity.

Fig. 4 shows the physical model of wheel and rail contact.



Fig. 4. Physical model of wheel and rail contact.

\section{Validation of the track model and wheel-rail interaction}

This section sets out some of the results obtained with the method presented in the paper. For the purposes of validation of the method, the results have been compared as a first step to those 
shown by $\mathrm{Wu}$ and Thompson $[16,17]$. It must be borne in mind that the initial receptances in this work feature a number of differences with respect to Wu's and Thompson's receptances, since they were obtained using different models, and thus the results of displacements after integration may be slightly different. At a second step the new model has been validated against Pieringer's results [27].

Fig. 5 shows the comparison between the results obtained in paper [17] and those calculated using the method described in this paper for displacements of the contact point on the wheel and the rail at a speed of $36 \mathrm{~m} / \mathrm{s}$, when there is no irregularity on the rail surface $(r=0)$. The track parameters are described in Table 1. The total weight of the vehicle corresponding to one wheel and the unsprung mass are, respectively, $W=100 \mathrm{kN}$ and $m_{w}=600 \mathrm{~kg}$; and the elastic spring constant is $C_{H}=93.7 \mathrm{GNm}^{-3 / 2}$. The first instant represented on the figures corresponds to movement over a sleeper, and shows movement over six spans of track. The Ss show the position of the sleepers. Both Fig. 5a and 5b show that the main harmonic in the displacements is that corresponding to the sleeper passage frequency, which in this case is $60 \mathrm{~Hz}$. It may also be observed that maximum dip at contact does not occur at exact midspan, but is displaced slightly to the right.

(a)


Fig. 5. Displacements of wheel (--) and rail (-) when speed $V=36 \mathrm{~m} / \mathrm{s}$ (a) obtained using the method described herein, (b) obtained through [17]. 
Fig. 6 shows the comparison between the displacements of wheel and rail when a wheel flat exists calculated in accordance with this work and the displacements obtained through [16]. The parameters used in this case are the same ones that those used for Fig. 5. This wheel flat was defined using expression (8) [14].

$$
r=\frac{d}{2}\left(1-\cos \left(2 \pi \frac{z}{l}\right)\right)
$$

The depth of the defect $(d)$ is $2 \mathrm{~mm}$, and its total length $(l)$ is $150 \mathrm{~mm}$. Fig. 6 shows superimposition of the displacements and representation of the defect. It may be observed that the individual results are extremely similar. Likewise, Fig. 7 shows the contact force between the wheel and the rail with this wheel flat. In both cases the force attains a maximum value above $500 \mathrm{kN}$ after the first loss of contact, and $200 \mathrm{kN}$ after the second.

(a)

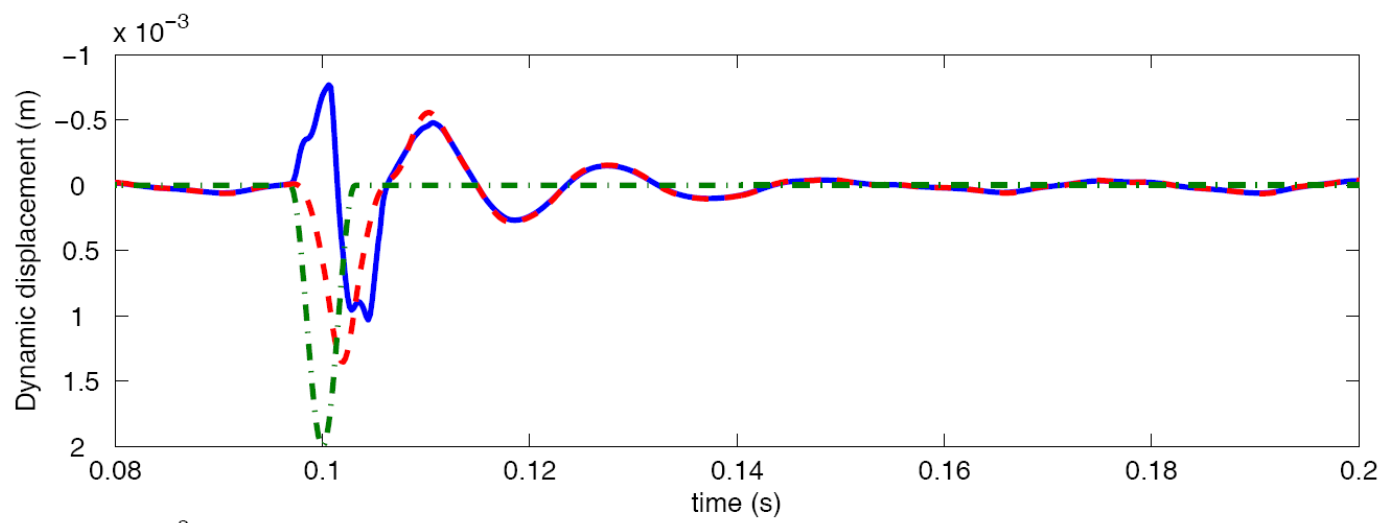

(b)

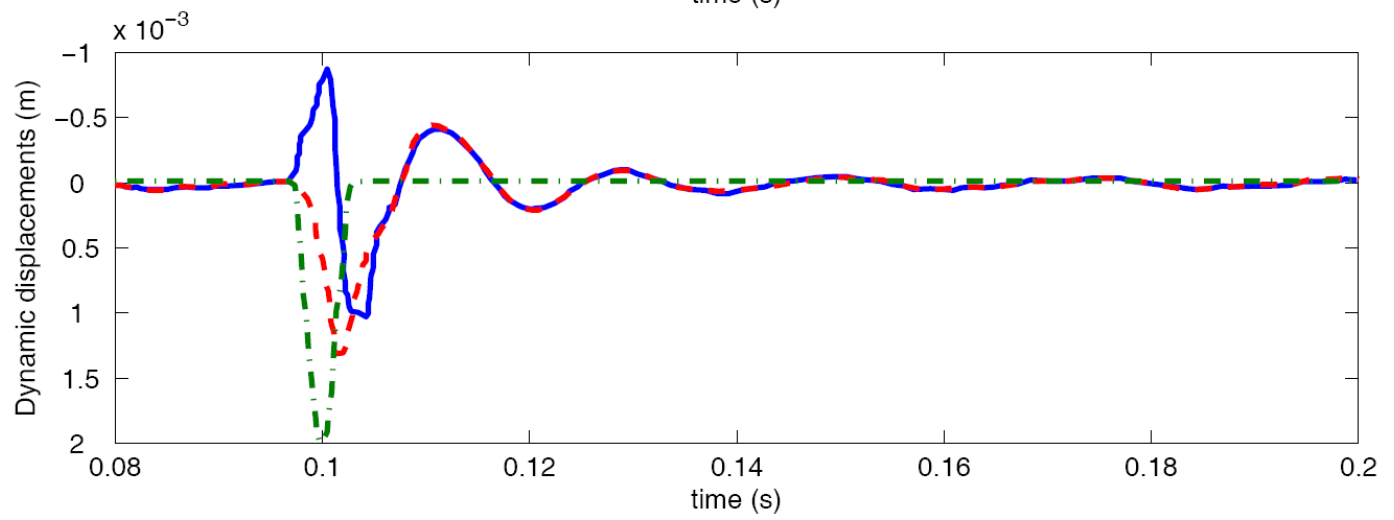

Fig. 6. Displacements of wheel (--) and rail (-) when speed $V=24 \mathrm{~m} / \mathrm{s}$ and a wheel flat (--) (a) obtained using the method described herein, (b) obtained through [16].

The results provided by the present model have also been compared to those published by Pieringer and Kropp in [27]. Such a model is also linear in what regards to the track and nonlinear in the contact, and has been applied to simulate wheel-rail interactions induced by wheel flats. Pieringer and Kropp, on their turn, compare their own results to those experimentally obtained by Johansson and Nielsen [28]. The results that follow have been 
compared to both, the ones of [27] and [28]. The parameters of this track and case study are shown in Table 2.

(a)

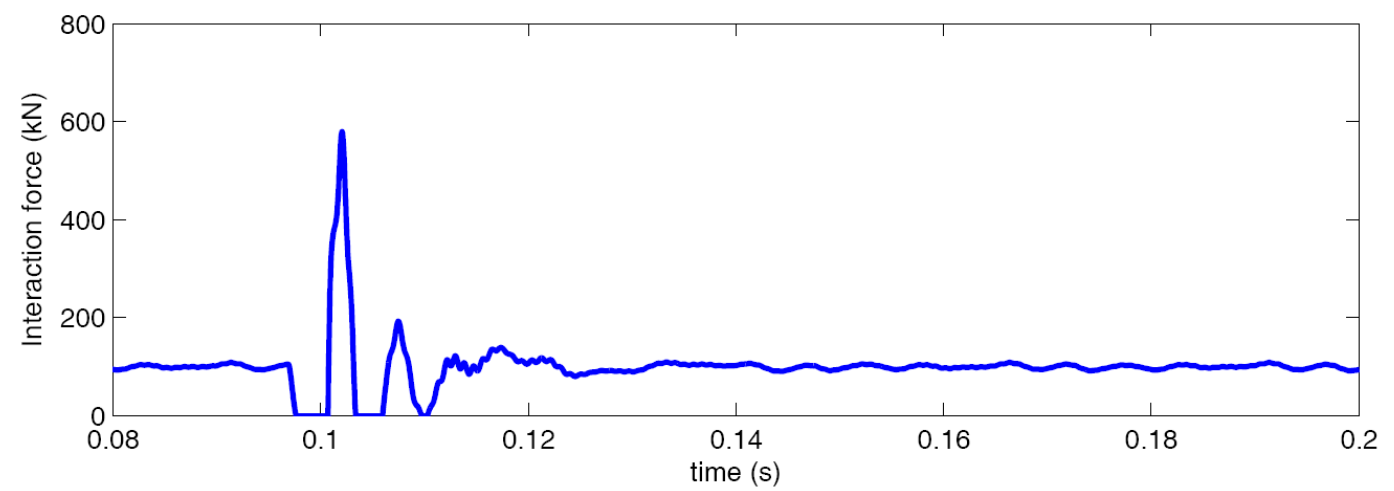

(b)

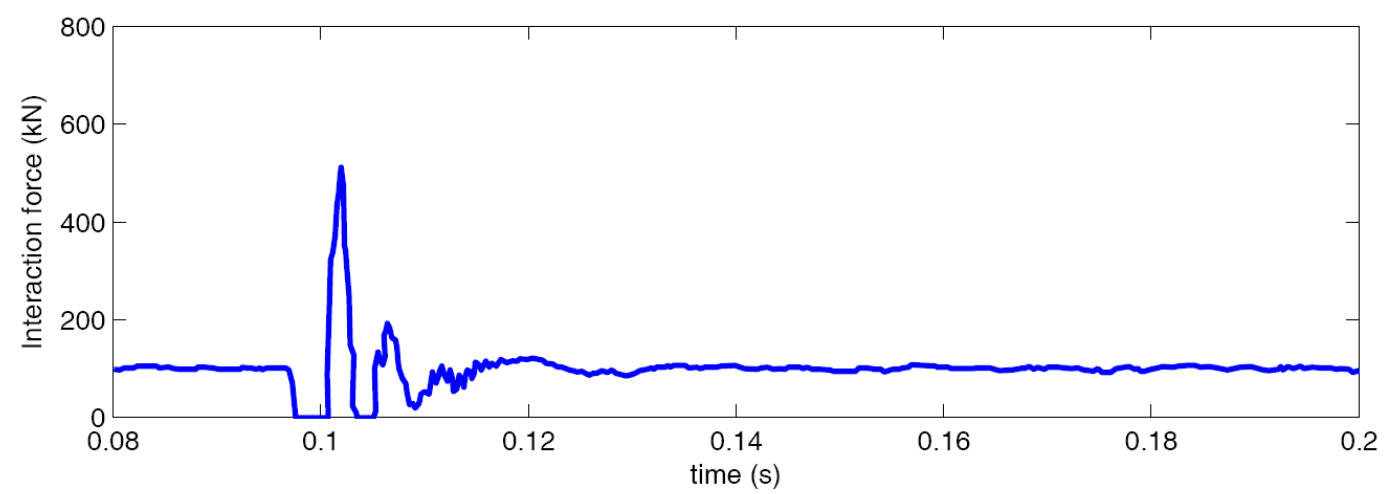

Fig. 7. Force of wheel and rail when speed $V=24 \mathrm{~m} / \mathrm{s}$ and a wheel flat (a) obtained using the method described herein, (b) obtained through [16].

Table 2: Track and vehicle parameters as described in [27]

\begin{tabular}{|l|c|}
\hline Pad stiffness $(\mathrm{kN} / \mathrm{mm})$ & 120 \\
\hline Pad damping $(\mathrm{kNs} / \mathrm{m})$ & 16 \\
\hline Ballast stiffness $(\mathrm{kN} / \mathrm{mm})$ & 140 \\
\hline Ballast damping $(\mathrm{kNs} / \mathrm{m})$ & 165 \\
\hline Sleeper mass $(\mathrm{kg})$ & 250 \\
\hline Sleeper spacing $(\mathrm{m})$ & 0.65 \\
\hline Rail & $60 \mathrm{E} 1$ \\
\hline Sprung mass $\mathrm{W}(\mathrm{t})$ & 12 \\
\hline Unsprung mass $(\mathrm{kg})$ & 592.5 \\
\hline Wheel radius $(\mathrm{m})$ & 0.45 \\
\hline
\end{tabular}

Fig. 8 shows the maximum contact forces produced by the wheel flat as defined in [27], comparing the experimental results from [28], the numerical results obtained by Pieringer [27] 
and the ones obtained by the model presented in this paper. The wheel flat has been defined according to expression (8), with $d=0.9 \mathrm{~mm}$ and $l=0.1 \mathrm{~m}$.

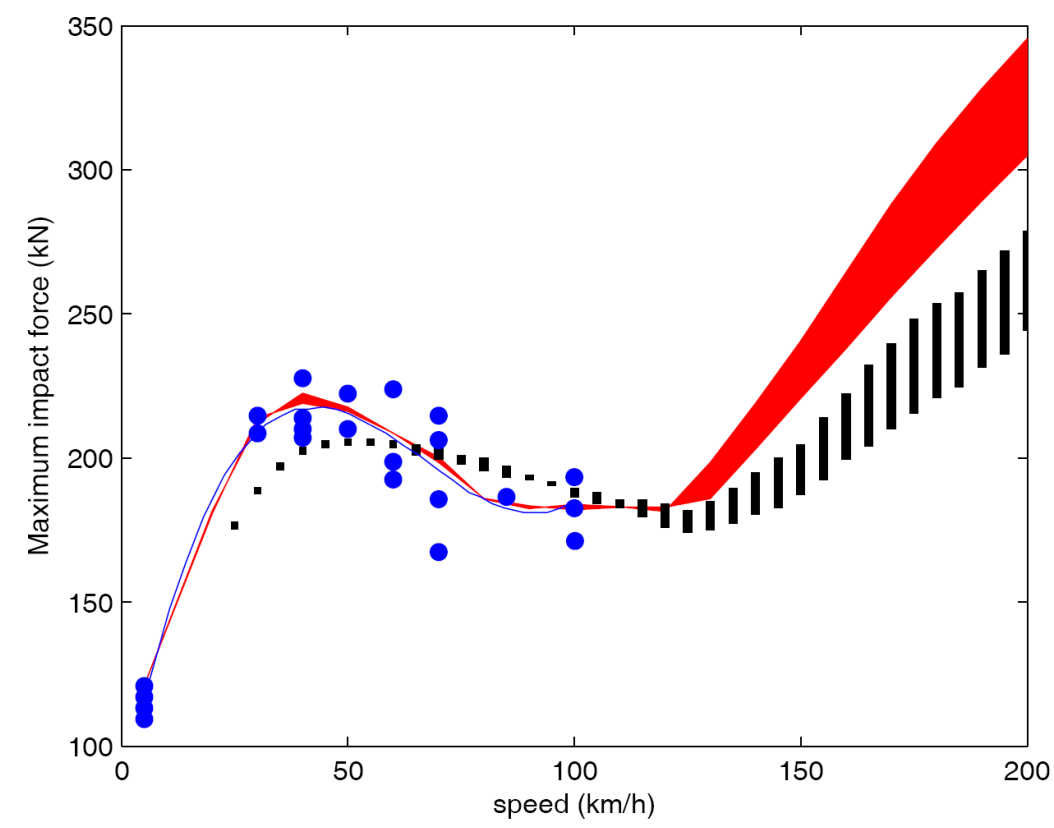

Fig. 8. Comparison between the maximum forces measured experimentally by Johansson [28] (measured values: - in blue, and curve fitted to their experimental results: continuous blue line); Pieringer's numerical results [27] ( $\cdot$ in black); and results provided by the new model (in red).

The experimental results by Johanson and Nielsen are shown as discrete values for several vehicle speeds, together with the curve fit. Both Pieringer's results and the ones obtained with the present model are shown for each speed as a vertical segment or as a band, respectively, taking account of the maximum and minimum values as a result of the position of the wheel flat relative to the sleeper. It is shown that for speeds up to $100 \mathrm{~km} / \mathrm{h}$ the results of the new model are extremely similar to the curve by Johanson and Nielsen fitted to their experimental results. And, despite the large conceptual differences between Pieringer's model and the model presented in this paper, both lead to results with reasonably similar trends.

Fig. 9 shows wheel and rail displacements when the wheelset, with the same wheel flat as before, runs at $50 \mathrm{~km} / \mathrm{h}$ along the same track. The sleeper is located al $\mathrm{x}=0$. Fig. 9 a shows the results obtained by Pieringer in [27] and Fig. 9b shows the results provided by the method developed. Fig. 10 compares wheel-rail contact forces, with the same criteria as above. As it can be seen the results are reasonably similar for both forces and displacements, despite the great differences between both models.

The differences between both results of the forces are at distance $0.08 \mathrm{~m}$, where the wheel flat starts, and a singular variation at distance $0.18 \mathrm{~m}$, where the wheel flat ends. The beginning and 
ending of the wheel flat is indicated with vertical dashed lines in Figs. 9 and 10. Both differences are due to the dissimilar contact model used by each method.

(a)

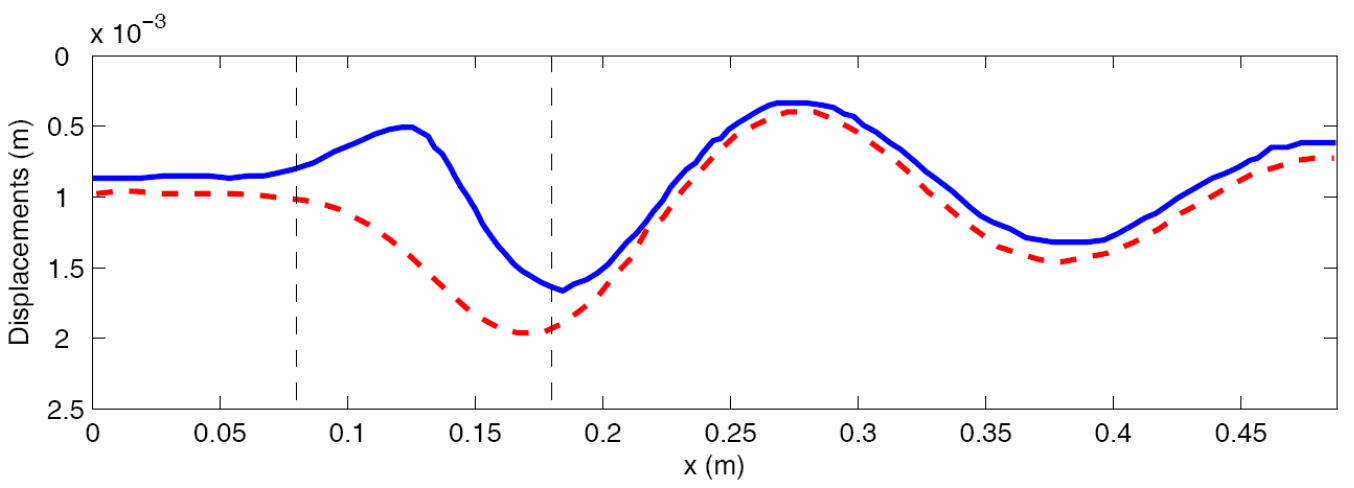

(b)

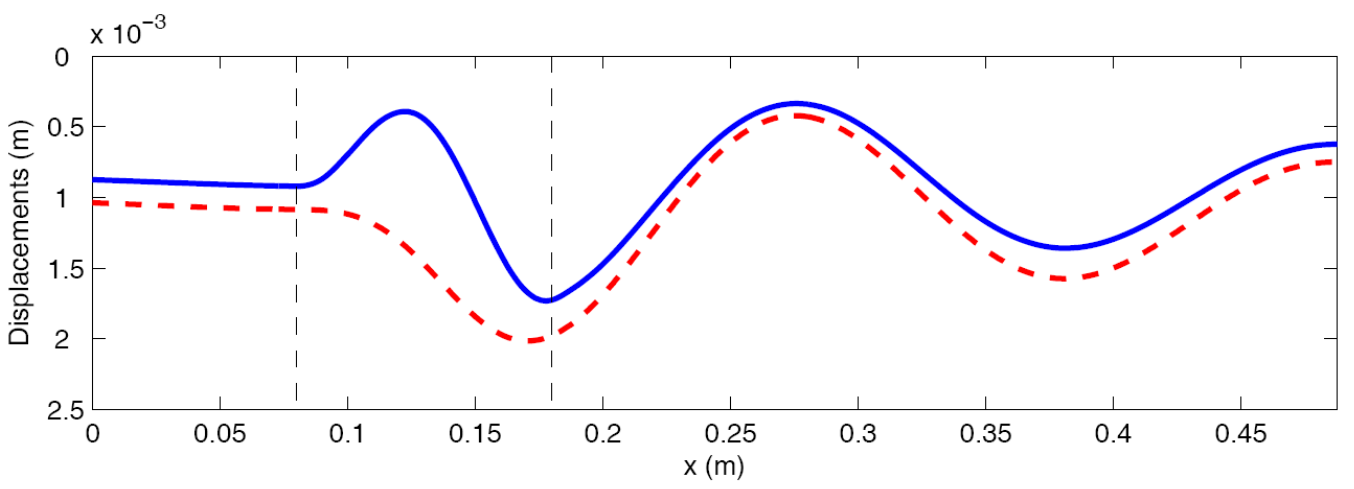

Fig. 9. Wheel (--) and rail (-) displacements when the wheel flat runs at $50 \mathrm{~km} / \mathrm{h}$ : (a) results by Pieringer and (b) results of the new model.

(a)

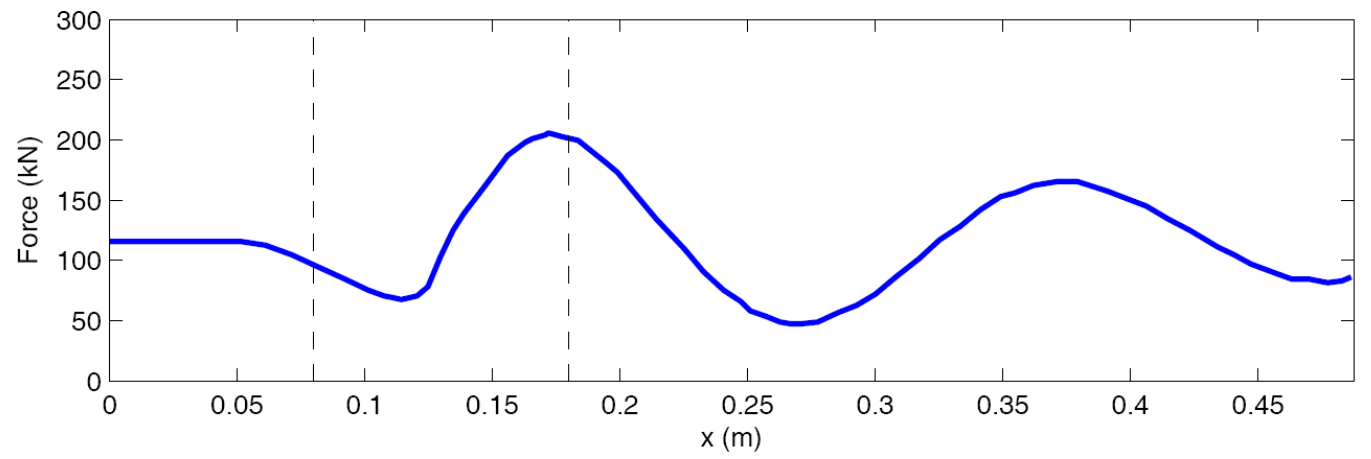

(b)

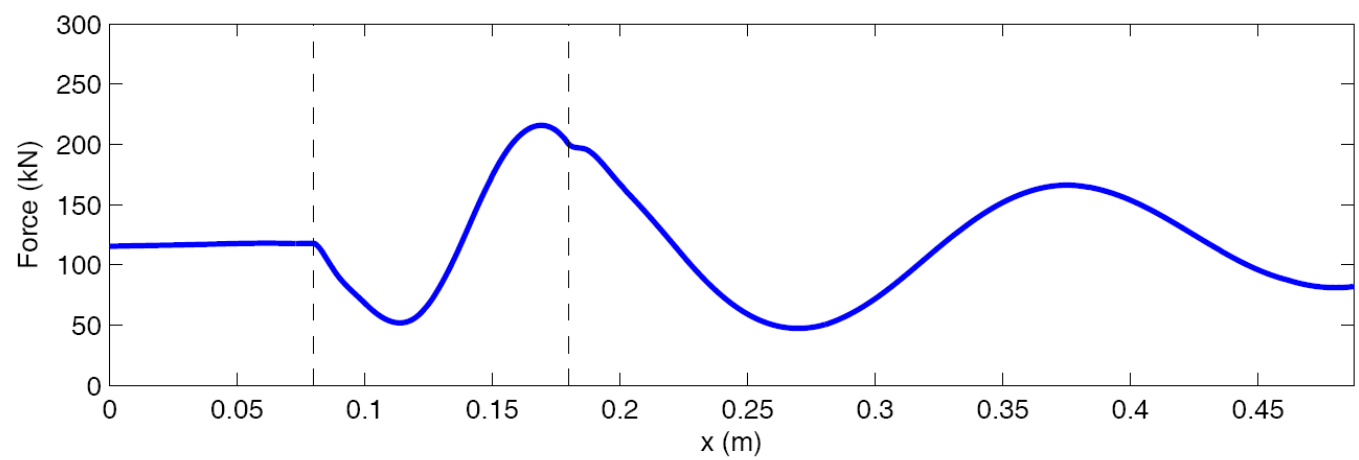


Fig. 10. Comparison between forces produced by a wheel flat at $50 \mathrm{~km} / \mathrm{h}$ obtained by: (a) results by Pieringer and (b) results of the new method.

\section{Application of the method for modelling forces and displacements on movement over a weld}

Rail welds constitute one of the critical features of continuous welded rail (CWR). Depending on the quality of the weld after grinding, large dynamic loads may arise as the wheelset moves over the track, and even loss of contact and impacts in certain cases, when the irregularity emerging from the weld is particularly large. Repeated movement of wheelsets over the welds may cause cracks in the rail due to fatigue, and this can lead to breakage of the rail, a particularly dangerous occurrence on the outside rail through a bend, since it could derail the train.

Several rail infrastructure authorities are concerned with tendencies in weld surfaces, which show dips after a number of years. As the wheelsets move over the rails, this effect is detected as an impact similar to that caused by a wheelset moving over a flanged joint.

This section shows some of the results obtained for the track previously fitted using the method set out herein, at different speeds, when the wheelset moves over the irregularity emerging from the weld.

The method developed herein is particularly suited to this type of calculation, since it reproduces the behaviour of the track extremely accurately, including the effect of the periodic excitation when the vehicle moves over the sleepers, and the computational outlay of each simulation is minimal.

Several welding geometry defects have been published as, for example, in [1, 29-31]. In the present work the first welding geometry defect shown by Steenbergen in [31] has been chosen. It is a welding experimentally measured by the author. A second welding geometry defect can be found as well in [31], although it has not been used in this work because it exceeds the limits described in the European Standard EN 14730-2 [3], which specifies the geometric characteristics that rail welds must verify after grinding to be acceptable. Fig. 11 shows the selected welding shape. In this dip the filtering effect due to the contact patch has already been considered, and a continuous smoothing of five points (taking a $25 \mathrm{~mm}$ length) has been performed.

Figs. 12 to 16 show the results of the wheel passing along the track described in Table 1, over a welding irregularity as described in Fig. 11, being the dip located $10 \mathrm{~cm}$ before the sleeper. The total weight of the vehicle corresponding to one wheel and the unsprung mass are, respectively, $W=100 \mathrm{kN}$ and $m_{w}=600 \mathrm{~kg}$; and the elastic spring constant is $C_{H}=93.7 \mathrm{GNm}^{-3 / 2}$.

Fig. 12 shows the displacements of the wheel and rail contact points, the contact force generated when the wheel is moving at $40 \mathrm{~km} / \mathrm{h}$, and the rail surface irregularity at the weld. It 
should be observed that the separation between the wheel and rail displacements is caused by deformation on the contact due to the weight of the vehicle borne by the wheel. The displacements' y-axis is inverted because displacements have been taken as positive when a dip occurs, and as negative if this is not the case. Displacement of the rail surface has been represented relative to the longitudinal geometry of the rail including the defect, in order to appraise at what points in space loss of contact may occur (as it may happen for certain cases of abnormal defects) and the impact when contact is retrieved. In the space before the weld defect, the figure above to the right shows the influence of movement over the sleeper, which gives the forces and displacements a periodic value rather than a constant value, with a spatial period defined by the sleeper spacing $(0.6 \mathrm{~m})$. The figure above has been enlarged in the area containing the weld for a better view of the forces and displacements in that area, and has been represented on the figure below.

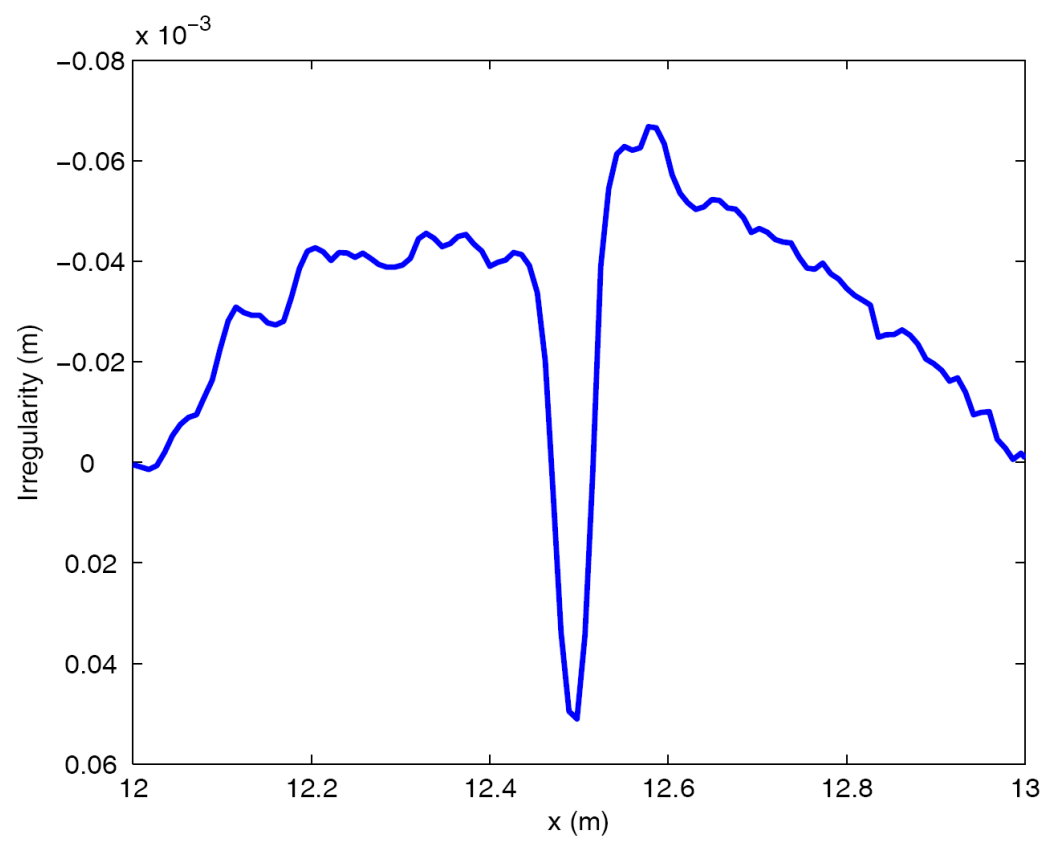

Fig. 11. Welding shape used [31].

At the beginning of the irregularity wheel and rail displacements are low, due to the fact that the first part of the welding defect geometry is a slight and smooth elevation of the rail surface. When the wheel commences the descent, as a result of the dipping on the rail surface in the central area of the rail defect, the force falls very slightly. After this decrease, there is an increase in the force during the defect's upward ramp, leading to a contact force maximum $20 \%$ above its average value. This is approximately twice the fluctuation of the force due to the wheelset passage over sleeper and midspan, which, on its turn, is approximately $10 \%$ of the static force supported by the wheel due to the vehicle weight. 
These figures also show that the excitation after the impact lasts over a very short space, and that the effect of passage over the weld disappears almost as the wheel reaches the end of the irregularity.

In the case of Fig. 13, where the results of the simulation at a running speed of $60 \mathrm{~km} / \mathrm{h}$ are shown, forces reach $134 \mathrm{kN}$. Given the higher speed, the duration of the excitation after passing over the welding defect is also longer, although it still remains very low.

(a)

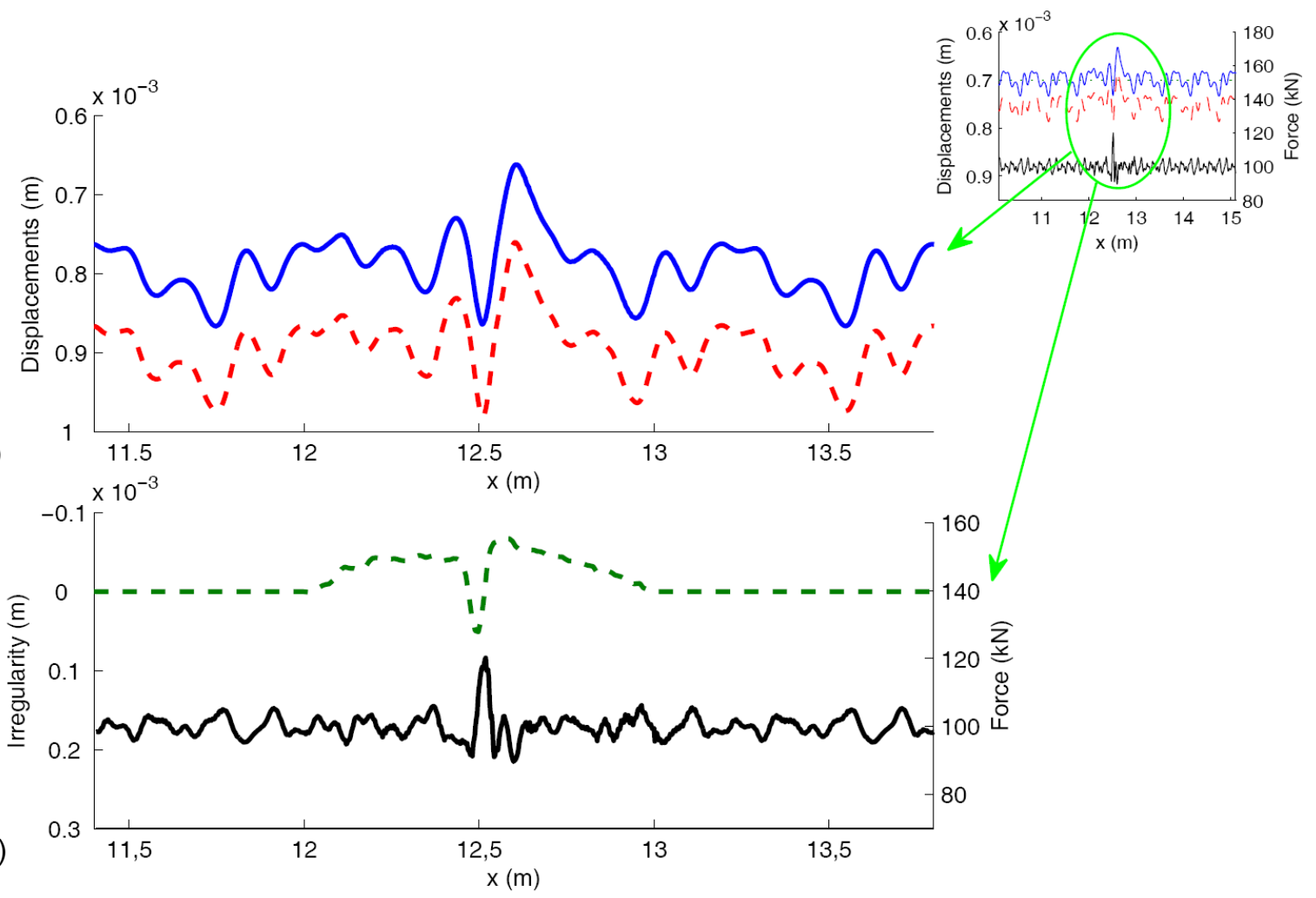

Fig. 12. (a) Displacements of wheel (--) and rail (-) at contact. (b) Irregularity (--) and contact forces (-) for a speed of $40 \mathrm{~km} / \mathrm{h}$. 
(a)


Fig. 13. (a) Displacements of wheel (--) and rail (-) at contact. (b) Irregularity (--) and contact forces (-) for a speed of $60 \mathrm{~km} / \mathrm{h}$.

Fig. 14 shows displacements and forces calculated for a running speed of $80 \mathrm{~km} / \mathrm{h}$. In this case the contact force reaches a maximum value of circa $144 \mathrm{kN}$, i.e. $44 \%$ above the static force due to the weight of the vehicle on the wheel. 
(a)
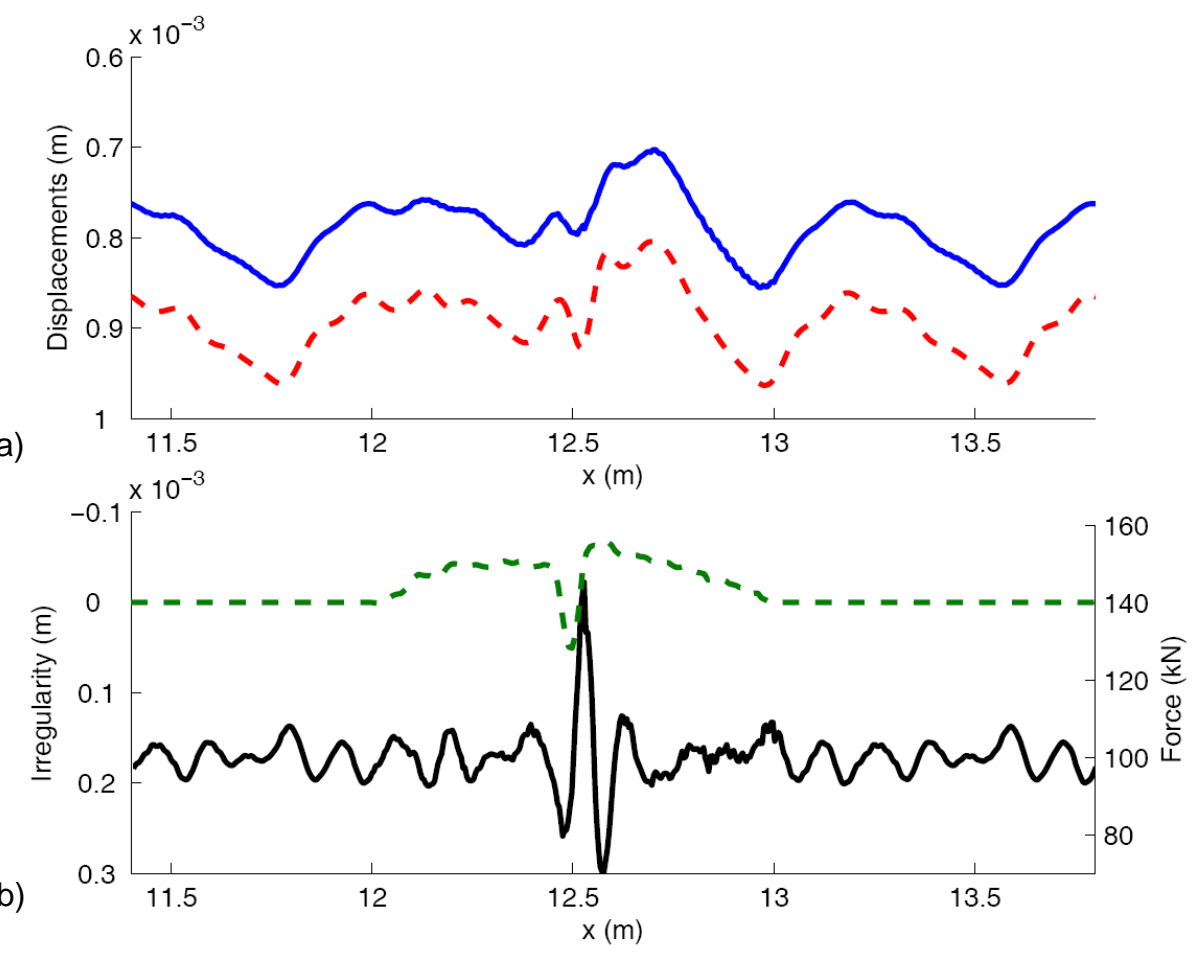

Fig. 14. (a) Displacements of wheel (--) and rail (-) at contact. (b) Irregularity (--) and contact forces (-) for a speed of $80 \mathrm{~km} / \mathrm{h}$.

Fig. 15 shows the contact forces for all three speeds. For these speeds, this track, and this location of the welding dip, it can be observed that the maximum forces in the contact increase with speed. It is also shown that the effect of passage over the weld is maintained over a greater distance when the speed is increased.

Parametric excitation has a significant importance in wheel-rail interactions, as already mentioned. The sleeper positioning considerably affects the dynamic wheel-rail interaction, and the model developed is able to take this into account. Therefore this capability has been used to quantify the influence on the wheel-rail forces of the defect position relative to the sleeper, in a similar way to what was mentioned previously in relation to the wheel flat positioning relative to the sleeper.

Fig. 16 shows the comparison between calculated forces when the welding is located at different positions. These results have been obtained for a wheelset running at a speed of 80 $\mathrm{km} / \mathrm{h}$ on a track whose receptances are the ones shown in Fig. 1. In this figure line $0 \mathrm{~L}$ corresponds to the welding located at the rail section above a sleeper, $0.25 \mathrm{~L}$ is the response when the welding is located at a quarter span distance from that sleeper, $0.5 \mathrm{~L}$ when the defect is just at midspan, and $0.75 \mathrm{~L}$ is the response if the welding defect is at three quarter span from the previous sleeper. 




Fig. 15. Comparison of contact forces for: speed $40 \mathrm{~km} / \mathrm{h} \mathrm{(--),} \mathrm{speed} 60 \mathrm{~km} / \mathrm{h}(-)$, and speed $80 \mathrm{~km} / \mathrm{h}(--)$.

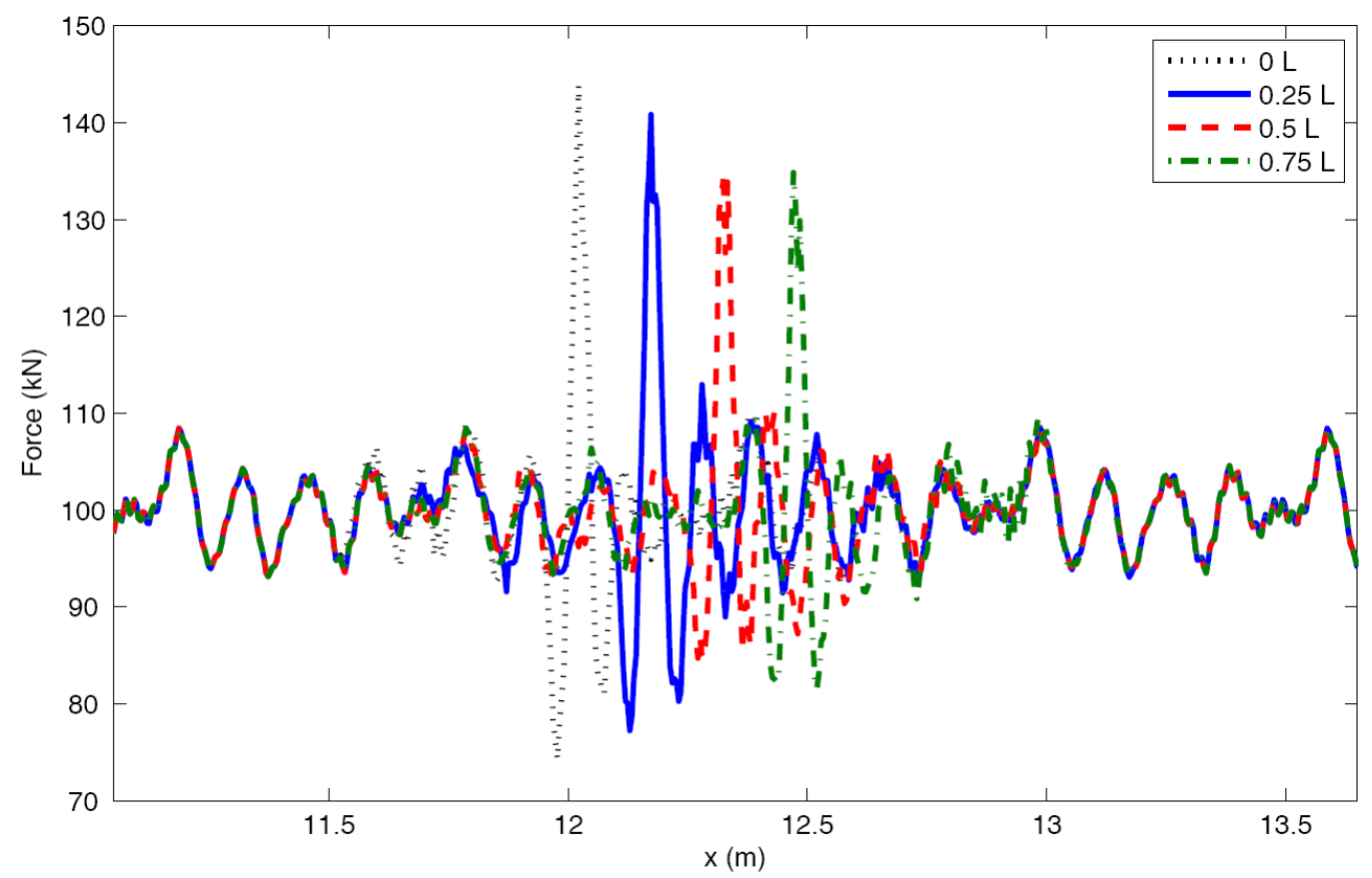

Fig. 16. Contact forces from welding defects at different positions within a span.

It can be seen that the largest forces appear when the welding defect is at a rail section located above the sleeper, reaching for this type of track and vehicle a maximum force of 144 
$\mathrm{kN}$, whereas the minimum forces appear when the welding defect is at midspan, reaching in this case $134 \mathrm{kN}$.

These forces are higher than usual. After a comparison of the forces obtained for different track parameters, it is concluded that in the present case the track is considerably stiff, leading therefore to such high forces. Slight reductions in track stiffness provoke the maximum forces to decrease up to $10 \mathrm{kN}$. For the case of the track described in [31] and with the same dip as before, even though the present model and the model described in that paper are based on completely different assumptions, the maximum forces provided by the present model for a speed of $140 \mathrm{~km} / \mathrm{h}$ are lower than the ones published in that paper: $125,7 \mathrm{kN}$ of total maximum force with the present method, against approximately $129,5 \mathrm{kN}$ of total maximum force $(17 \mathrm{kN}$ of dynamic force) obtained in [31].

\section{Conclusions}

A method has been developed to improve and facilitate the passage of track models in the frequency domain to models in the spatial domain. This can be a very interesting procedure when an extremely precise model in the frequency domain is available which, in comparison to the usual spatial-domain models, can take account to a high degree of accuracy of the track's dynamic behaviour, including deformation of the rail section and the propagation of elongation, bending and buckling waves, and the intention is to make use of the advantages of this model with respect to more simplified models in the spatial domain. Moreover, the model produced by this method is very simple, with very few degrees of freedom, thus compensating the time required for passage to the spatial domain with a substantial reduction of simulation time.

The method developed is based on the receptances obtained using the model in the frequency domain on track sections in as close proximity as is desired, and this secures a model in the spatial domain that adjusts to these receptances. To this end the process is based on application of the rational fraction polynomials method, with a weight vector at each frequency of the fitting optimised using genetic algorithms, in order to obtain the best possible fit of track receptances. The result of this fitting is a transfer function the frequency response of which represents a good fit for the track receptance, and which can be transformed into a system of ordinary differential equations by applying the inverse Laplace transform. This fitting is repeated for different sections of the track span, and the result obtained is a system of equations in the spatial domain which varies along the track span. The method also guarantees the stability of the resultant system in the spatial domain and the fitting coefficients set, which facilitate integration of the system of equations.

The method has been validated with the results obtained by $\mathrm{Wu}$ and Thompson $[16,17]$ and by Pieringer [27]. The model is applicable to most wheel-rail dynamic interaction problems, and 
it has been applied to the study of the displacements and forces that emerge when the wheelset moves over a weld. It has been possible to compare the increase in the contact forces produced by the welding defect with the increase in such forces as a result of the parametric excitation due to the discrete support. Welding defects complying with international standards have been studied and contact forces and displacements have been calculated at different speeds. The model has been used to quantify the influence of the welding being over a sleeper, on the centre of the span or at intermediate locations.

\section{Acknowledgements}

The authors thank the Spanish Research Ministry MICINN for their funding through contract TRA2010-18386 including the FEDER funds of the European Union, as well as the Basque Government for its financial assistance through IT-453-10 and for Research Grant BFI08.172. They also thank the Basque Railway Infrastructure Manager ETS/RFV and Metro Bilbao for their assistance and valuable suggestions.

\section{References}

[1] M. J. M. M. Steenbergen, C. Esveld. Rail weld geometry and assessment concepts, Proceedings of the IMechE, Part F: Journal of Rail and Rapid Transit, 2006, 220 (F3), 257-271.

[2] M. J. M. M. Steenbergen, C Esveld. Relation between the geometry of rail welds and the dynamic wheel - rail response: numerical simulations for measured welds, Proceedings of the IMechE, Part F, Journal of Rail and Rapid Transit, 2006, 220 (F4), 409-424.

[3] EN 14730-2:2006. Railway applications. Track. Aluminothermic welding of rails. Qualifications of aluminothermic welders, approval of contractors and acceptance of welds.

[4] A. V. Vostroukhov, A. V. Metrikine. Periodically supported beam on a visco-elastic layer as a model for dynamic analysis of a high-speed railway track, International Journal of Solids and Structures. 40 (2003) 5723-5752.

[5] M. F. M. Hussein, H. E. M. Hunt. Modelling of floating-slab tracks with continuous slabs under oscillating moving loads, Journal of Sound and Vibration. 297 (2006) 37-54.

[6] I. Gómez, E. G. Vadillo. An analytical approach to study a special case of booted sleeper track rail corrugation, Wear. 251 (2001) 916-924. 
[7] I. Gómez, E. G. Vadillo. A linear model to explain short pitch corrugation on rails, Wear. 255 (2003) 1127-1142.

[8] J. Gómez, E. G. Vadillo, J Santamaría. A comprehensive track model for the improvement of corrugation models, Journal of Sound and Vibration. 293 (2006) 522-534.

[9] O. Oyarzabal, J. Gómez, J. Santamaría, E. G. Vadillo. Dynamic optimization of track components to minimize rail corrugation, Journal of Sound and Vibration. 319 (2009) 904-917.

[10] T. Mazilu. Green's functions for analysis of dynamic response of wheel/rail to vertical excitation, Journal of Sound and Vibration. 306 (2007) 31-58.

[11] T. Mazilu, M. Dumitriu, C. Tudorache, M. Sebeşan. Using the Green's functions method to study wheelset/ballasted track vertical interaction, Mathematical Computer Modelling. 54 (2011) 261-279.

[12] T. X. Wu, D. J. Thompson. Theoretical investigation of wheel/rail non-linear interaction due to roughness excitation, Vehicle System Dynamics. 34 (2000) 261-282.

[13] T. X. Wu, D. J. Thompson. A hybrid model for the noise generation due to railway wheel flats, Journal of Sound and Vibration. 251 (2002) 115-139.

[14] T. X. Wu, D. J. Thompson. On the impact noise generation due to a wheel passing over rail joints, Journal of Sound and Vibration. 267 (2003) 485-496.

[15] T. X. Wu, D. J. Thompson. Wheel/rail non-linear interactions with coupling between vertical and lateral directions, Vehicle System Dynamics. 41 (2004) 27-49.

[16] T. X. Wu, D. J. Thompson. On the parametric excitation of the wheel/track system, Journal of Sound and Vibration. 278 (2004) 725-747.

[17] T. X. Wu, D. J. Thompson. On the rolling noise generation due to wheel/track parametric excitation, Journal of Sound and Vibration. 293 (2006) 566-574.

[18] T. X. Wu. Parametric excitation of wheel/track system and its effects on rail corrugation, Wear. 265 (2008) 1176-1182.

[19] G. Bonin, G. Cantisani, M. Carbonari, G. Loprencipe, A. Pancotto, Railway traffic vibrations: generation and propagation - Theoretical aspects, 4th International SIIV Congress Palermo (Italy). (2007). 
[20] G. Bonin, G. Cantisani, M. Carbonari, G. Loprencipe, A. Pancotto, Railway traffic vibrations: generation and propagation - Use of computational models, 4th International SIIV Congress - Palermo (Italy). (2007).

[21] M.H. Richardson, D. L. Formenti, Parameter estimation from frequency response measurements using rational fraction polynomials. (1982).

[22] F. H. Raven, Automatic control Engineering, 4th ed. ed., McGraw-Hill, New York, 1987.

[23] R. Taghipour, T. Perez, T. Moan. Hybrid frequency-time domain models for dynamic response analysis of marine structures, Ocean Engineering. 35 (2008) 685-705.

[24] The MathWorks Inc., Optimization Toolbox ${ }^{\mathrm{TM}}$ User's Guide, 2010.

[25] The MathWorks Inc., Global Optimization Toolbox User's Guide, 2010.

[26] The MathWorks Inc., Signal Processing Toolbox ${ }^{\text {TM }}$ User's Guide, 2010.

[27] A. Pieringer, W. Kropp, A fast-time-domain model for wheel/rail interaction demonstrated for the case of impact forces caused by wheel flats. Proceedings of Acoustics '08, Paris, France, June 29-July 4, 2008.

[28] A. Johansson, J. C. O. Nielsen. Out-of-round railway wheels-wheel-rail contact forces and track response derived from field tests and numerical simulations, Proceedings of the Institution of Mechanical Engineers, Part F: Journal of Rail and Rapid Transit. 217 (2003) 135146.

[29] W. Li, G. Xiao, Z. Wen, X Xiao, X. Jin. Plastic deformation of curved rail at rail weld caused by train-track dynamic interaction, Wear. 271 (2011) 311-318.

[30] Z. Wen, G. Xiao, X. Xiao, X. Jin, M. Zhu. Dynamic vehicle-track interaction and plastic deformation of rail at rail welds, Engineering Failure Analysis. 16 (2009) 1221-1237.

[31] M. J. M. M. Steenbergen. Quantification of dynamic wheel-rail contact forces at short rail irregularities and application to measured rail welds, Journal of Sound and Vibration. 312 (2008) 606-629. 